\title{
Intervenção nutricional no tratamento cirúrgico da obesidade mórbida: resultados de um protocolo diferenciado
}

\author{
Nutritional intervention in the surgical treatment for \\ morbid obesity: results of a differentiated protocol
}

Magda Rosa Ramos da CRUZ ${ }^{1}$

Ivone Mayumi Ikeda MORIMOTO²

\section{RE S U M O}

Com o objetivo de verificar os resultados de um protocolo diferenciado, no que se refere a especificidades no trabalho de pré-operatório e à evolução para dieta sólida a partir da $3^{a}$ semana de pós-operatório, foram estudados $100 \%$ dos pacientes atendidos durante o período de abril de 2000 a agosto de 2001 . Um total de 50 pacientes que apresentavam variação do Índice de Massa Corporal de $40 \mathrm{~kg} / \mathrm{m}^{2}$ a $55 \mathrm{~kg} / \mathrm{m}^{2}$ foram avaliados. Os resultados obtidos, tanto no pré quanto no pós-operatório, foram positivos, mostrando que $60 \%$ dos pacientes no pré-operatório reduziram seu peso. No pós-operatório, até o $21^{\circ}$ dia de alimentação, os resultados foram favoráveis quanto à perda de peso e mostraram baixa incidência das principais complicações deste período, relatadas em literatura. Assim, enfatiza-se a importância da elaboração de um protocolo de atendimento nutricional que contemple e priorize aspectos de educação nutricional, preparo cuidadoso do paciente no pré-operatório e monitorização detalhada no pós-operatório.

Termos de indexação: obesidade mórbida, gastroplastia, terapia nutricional, perda de peso.

\section{A B S T R A C T}

The goal of this research is to verify the results of a differentiated protocol that refers to the particularities in the preoperative period and the evolution of the solid diet after the $3^{\text {rd }}$ week of the postoperative period.

\footnotetext{
1 Núcleo de Cirurgia e Tratamento da Obesidade Mórbida. Curso de Nutrição, Pontifícia Universidade Católica do Paraná. R. Imaculada Conceição, 1155, Prado Velho, 80215-901, Curitiba, PR, Brasil. Correspondência para/Correspondence to: M.R.R. CRUZ.

${ }^{2}$ Curso de Nutrição, Pontifícia Universidade Católica do Paraná, e do Centro Universitário Positivo, Curitiba, PR, Brasil.
} 
Between April 2000 and August 2001, 100\% of the attended patients were studied. A total of 50 patients, with BMI variation between $40 \mathrm{~kg} / \mathrm{m}^{2}$ and $55 \mathrm{~kg} / \mathrm{m}^{2}$ were observed. The obtained results in pre and postoperative periods were positive, and demonstrated that $60 \%$ of the patients reduced their weight. Until the $21^{\text {st }}$ day with oral feeding in postoperative period, patients demonstrated satisfactory results in terms of weight loss and low incidence of the main complications related to this period, as described in literature. Therefore, it is important to elaborate a Nutritional Attendance Protocol that contemplates and prioritizes nutritional education aspects, the preparation of the patient before surgery and detailed monitoring after surgery.

Index terms: obesity, morbid, gastroplasty, nutritional therapy, weight loss.

\section{N T R O D U Ç Ã O}

Segundo Garrido Júnior et al. ${ }^{1}$, a cirurgia para tratamento da obesidade grave vem sendo empregada há quase 50 anos. Iniciou-se na década de 1950 com operações que causavam má absorção, abandonadas no fim da década de 1970 pelos seus efeitos indesejáveis graves e freqüentes, tais como: diarréia, desidratação, vômitos, dor abdominal, problemas hepáticos e cirrose. A partir de então, passaram a predominar os procedimentos que limitam a ingestão de alimentos, seja pela simples restrição da capacidade do estômago, seja por sua divisão e anastomose ao jejuno proximal.

Dentre as técnicas cirúrgicas, a mais utilizada é a de Fobi-Capella, uma técnica mista, ou seja, restritiva e disabsortiva. Nesta, o estômago é separado em dois compartimentos por meio do uso de grampeadores. O estômago remanescente terá um volume de $30 \mathrm{~mL}$ a $50 \mathrm{~mL}$ e será ligado a um segmento do intestino delgado, enquanto a maior porção do estômago fica fora do trânsito alimentar².

O tratamento cirúrgico da obesidade justifica-se somente quando o risco de permanecer obeso exceder os riscos, a curto e longo prazo, da cirurgia bariátrica. Os critérios para a seleção do paciente incluem: índice de massa corporal (IMC) igual ou superior a $40 \mathrm{~kg} / \mathrm{m}^{2}$ ou acima de $35 \mathrm{~kg} / \mathrm{m}^{2}$ associado a comorbidades que possam ser reduzidas com a perda de peso; várias tentativas de perda de peso sem resultado e condições psicológicas para cumprir a orientação dietética no pós-operatório, o que deve ser avaliado com a ajuda do serviço de psicologia ${ }^{3,4}$.
A conclusão da cirurgia não finaliza o tratamento da obesidade, pelo contrário, é o início de um período de um a dois anos de mudanças comportamentais, alimentares e de exercícios, com monitoração regular de uma equipe multidisciplinar de profissionais da saúde.

Segundo Herrera et al. ${ }^{5}$, a cirurgia bariátrica feita pela técnica de Capella leva a uma perda total de $75 \%$ do excesso de peso no decorrer de um ano e é de extrema importância no tratamento da obesidade mórbida, por ser capaz de reduzir, ou até mesmo eliminar, as principais comorbidades relacionadas ao excesso de peso e, com isso, trazer melhorias na qualidade de vida do paciente.

Csendes et al. ${ }^{6}$ citam as enfermidades coronarianas, hipertensão, alterações da função da bomba cardíaca, diabetes melitus precoce, hipoventilação ou apnéia do sono e embolia pulmonar como as principais comorbidades relacionadas à obesidade grave. Outras doenças que podem estar associadas ao excesso de peso são: colelitíase, osteoartrite degenerativa, úlcera venosa, hérnia hiatal, inguinal e umbilical e câncer de útero, mama e cólon.

Cowan \& Buffington ${ }^{7}$ realizaram acompanhamento de 82 pacientes obesos mórbidos, dos quais 33\% eram hipertensos, 16\% eram diabéticos e 33\% apresentavam níveis elevados de glicemia de jejum e constataram que no pósoperatório de cirurgia bariátrica houve redução dos níveis de glicemia de jejum em todos os pacientes, a diabetes foi solucionada em 92,3\% dos casos e a pressão sangüínea foi reduzida 
significativamente após a perda de peso de aproximadamente $30 \%$ do peso total.

Valezi et al. ${ }^{8}$, em experiência de 200 casos, verificaram redução de peso de $43,5 \%$, em um período de 12 meses. Halverson ${ }^{9}$ define como resultado de sucesso uma perda acima de $50 \%$ do excesso de peso.

O protocolo de nutrição para atendimento dessa população envolve os seguintes períodos: pré-operatório, intra-hospitalar e pós-operatório.

Segundo Cronin \& Mac Donough ${ }^{10}$, o intuito do aconselhamento nutricional no período pré-operatório é o aumento do potencial de sucesso no pós-operatório e objetiva promover perda de peso inicial, reforçar a percepção do paciente de que a perda de peso é possível quando o balanço energético se torna negativo, identificar erros e transtornos alimentares, promover expectativas reais de perda de peso, preparar o paciente para a alimentação no pós-operatório e verificar o potencial do paciente para o sucesso da cirurgia.

Shikora ${ }^{2}$ afirma que um bom entendimento das mudanças da capacidade e função gástrica e das restrições dietéticas é decisivo para um ótimo resultado após a cirurgia. O plano de introdução gradual de alimentos deve ser apresentado com recursos que garantam a compreensão do paciente sobre a capacidade gástrica no pós-operatório, sobre desconfortos fisiológicos potenciais e conseqüências que podem ser experimentadas se o protocolo não for seguido. A responsabilidade do indivíduo no autocuidado é enfatizada durante esta fase.

Cronin \& Mac Donough ${ }^{10}$ ressaltam a importância do trabalho em equipe para relatar o progresso de cada paciente a todos os membros, de forma que somente pacientes que tenham resultado satisfatório sejam liberados para cirurgia.

Alguns autores tradicionalmente recomendam a prescrição de alimentação de consistência líquida restrita, evoluindo para líquida completa até a alta hospitalar, após a realização da cirurgia. A etapa seguinte envolve a introdução de alimentos pastosos para evitar distensão gástrica, ocorrendo a evolução para alimentação sólida somente após 12 semanas da realização da cirurgia, enfatizando-se a mastigação suficiente para os alimentos até que esses atinjam a consistência pastosa, para só então degluti-los 3,10. Martin et al. ${ }^{11}$ recomendam a manutenção de dieta líquida por seis semanas antes do início do processo de transição para dieta de maior consistência, enquanto Cronin \& Mac Donough ${ }^{10}$ enfatizam a necessidade de manutenção de dieta pastosa durante dois meses.

A literatura sobre o assunto demonstra uma grande incidência de complicações no pós-operatório como náuseas, vômitos, síndrome de Dumping, diarréia, constipação, obstrução gástrica, ruptura da linha de grampeamento e deficiência de alguns micronutrientes específicos, tais como: ácido fólico, ferro e vitamina $\mathrm{B}_{12}$, a qual justifica, mais uma vez, a necessidade de intervenção nutricional adequada $3,5,9$.

A elaboração do protocolo proposto para o Núcleo de Cirurgia e Tratamento da Obesidade Mórbida (NUCTROM), sediado na cidade de Curitiba, PR, considerou inicialmente as orientações de evolução de consistência relatadas em literatura, mas no contato diário com o paciente verificou-se a necessidade de algumas adaptações quanto ao tempo necessário de contato no pré-operatório, suficiente para realizar um trabalho efetivo, e quanto à evolução de consistência, de maneira a proporcionar um maior conforto para o paciente, sem causar, todavia, aumento na incidência de complicações no pós-operatório.

As adaptações acima citadas originaram um protocolo de nutrição diferenciado, contrapondo-se aos relatos existentes em literatura até o presente momento, principalmente quanto ao aspecto do período de tempo necessário para introdução de alimentos sólidos.

O presente trabalho teve como objetivo constatar os resultados de um protocolo de nutrição diferenciado, utilizado no tratamento 
cirúrgico da obesidade mórbida, em um único centro de tratamento.

\section{CASUÍSTICA E MÉTODOS}

Foram estudados 50 pacientes atendidos durante o período de abril de 2000 a agosto de 2001, no consultório de nutrição, com IMC variando entre $40 \mathrm{~kg} / \mathrm{m}^{2}$ e $55 \mathrm{~kg} / \mathrm{m}^{2}$. O encaminhamento para o atendimento nutricional teve como objetivo preparar o paciente para a cirurgia bariátrica pela técnica de Fobi-Capella, e acompanhar sua evolução no pós-operatório, seguindo o protocolo apresentado a seguir. Os registros das consultas iniciais e de acompanhamento, tanto no pré como no pós-operatório, foram analisados tomando-se como indicadores a perda de peso no pré-operatório, a freqüência de intolerância alimentar e de vômitos e a perda de peso nas 3 primeiras semanas de pós-operatório. Estes indicadores foram estabelecidos com a intenção de avaliar os resultados do protocolo proposto, sem a intenção de realizar um estudo comparativo, pois trata-se de um protocolo diferenciado não havendo, portanto, publicações de trabalhos semelhantes.

O protocolo foi aprovado pelo Comitê de Ética em Pesquisa dos Hospitais da Aliança Saúde PUCPR-Santa Casa, da cidade de Curitiba, PR.

\section{Protocolo de atendimento nutricional proposto}

\section{No pré-operatório}

Por meio da avaliação nutricional, detalhada no pré-operatório, obtêm-se informações importantes sobre o estilo de vida, hábitos alimentares e estado nutricional do paciente.

A avaliação do estado nutricional é feita a partir da análise de exames laboratoriais (hemograma completo, dosagem sanguínea de proteínas totais e frações, ferro sérico, vitamina $B_{12}$, ácido fólico e zinco, além de colesterol total e frações, triglicerídeos, creatinina, ácido úrico e uréia), IMC atual, peso habitual, presença de doenças associadas, história mórbida familiar (HMF), pregressa (HMP), atual (HMA) e anamnese alimentar, a qual inclui métodos de freqüência de consumo alimentar e recordatório 24 horas $^{3,9,10}$.

Somente após a avaliação nutricional, pode-se afirmar se há ou não indicação de cirurgia para o paciente. Vale ressaltar que todos os profissionais da equipe (cirurgião, nutricionista, fisioterapeuta, psicóloga e psiquiatra) reúnem-se para discussão de casos e emitem laudos, liberando o paciente para a cirurgia. Estes laudos são entregues pelo próprio paciente ao médico cirurgião, pois sem a apresentação dos mesmos não há prosseguimento do processo de encaminhamento para a cirurgia.

Passando essa fase de levantamento de dados, utiliza-se o Guia Alimentar da Pirâmide dos Alimentos, publicado em 1992 pelo Departamento de Agricultura dos E.U.A. como instrumento para ensinar ao paciente as práticas alimentares saudáveis $^{12}$. O nutricionista preenche uma pirâmide alimentar em branco com a alimentação relatada no recordatório de 24h; a seguir, explica ao paciente os princípios da alimentação equilibrada, segundo esse guia, e pede que ele compare a pirâmide preenchida com a pirâmide ideal. O paciente emite então a sua opinião sobre as providências a serem tomadas para melhorias na sua alimentação. O estabelecimento de metas é realizado pelo próprio paciente, de acordo com as suas possibilidades, havendo participação do nutricionista como facilitador do processo. Os benefícios da alimentação equilibrada no pré-operatório são esclarecidos ao paciente, quais sejam: melhores resultados no pós-operatório, traduzidos em uma melhor cicatrização da ferida cirúrgica e menor incidência de complicações nutricionais tardias.

Após um período de 15 dias, ocorre a segunda consulta. Nesta, verifica-se quanto das 
metas estabelecidas foram efetivamente alcançadas, ou seja, se houve melhora na qualidade da sua alimentação conforme a proposta estabelecida pelo guia da pirâmide alimentar, e realiza-se, também, nova pesagem para verificar se houve redução de peso. Nessa consulta, é apresentado ao paciente o esquema alimentar que será utilizado nas primeiras semanas de pós-operatório, para que haja tempo para esclarecimento de todas as suas dúvidas, o que influencia nos aspectos de segurança e confiança em relação à cirurgia.

Outro fator observado neste momento é a mastigação do paciente e a distribuição de suas refeições durante o dia. O paciente é estimulado a fazer 6 refeições ao dia, adaptando-as ao seu ritmo de vida e aproximando-se do esquema de refeições necessário no pós-operatório.

A família é envolvida no processo devido ao seu papel primordial no apoio ao paciente no pós-operatório, sendo este estimulado a comparecer na segunda consulta com um ou mais familiares que possam ajudá-lo.

\section{Intra-hospitalar}

O cirurgião, ou o residente, prescreve a dieta considerando o dia de alimentação via oral, no pós-operatório, da seguinte maneira: " $1^{\circ} \mathrm{PO}$ gastroplastia" ou " $2^{\circ}$ PO gastroplastia" com a finalidade de diferenciar a prescrição em relação às dietas líquidas sem limitação de volume. Ao verificar este registro no Mapa Diário de Dietas, os nutricionistas identificam esses pacientes e garantem o fornecimento de alimentação adequada.

A dieta padronizada é de consistência líquida, em horários regulares, respeitando o volume, que não deverá ultrapassar $50 \mathrm{~mL}$ por refeição, para não provocar náuseas e vômitos ${ }^{3,10}$.

A dieta padronizada para o primeiro e o segundo dias de alimentação após a cirurgia (Tabela 1) tem como objetivo facilitar o processo de digestão e posterior esvaziamento gástrico, além de impedir que resíduos possam aderir à região grampeada, sendo oferecidos apenas líquidos coados. O leite é introduzido apenas no segundo dia de alimentação, caso o paciente

Tabela 1. Dieta padronizada para o período de internação.

\begin{tabular}{|c|c|c|c|}
\hline \multicolumn{4}{|c|}{ Primeiro dia de dieta pós-gastroplastia ( $\left.1^{\circ} \mathrm{PO}\right)$} \\
\hline Horário & Alimento & Quantidade & Kcal \\
\hline $8 \mathrm{~h}$ & Chá de ervas com adoçante Diet & $50 \mathrm{~mL}$ & 1 \\
\hline $10 \mathrm{~h}$ & Suco de laranja coado e sem adoçar & $50 \mathrm{~mL}$ & 32 \\
\hline $12 \mathrm{~h}$ & Sopa líquida restrita & $50 \mathrm{~mL}$ & 25 \\
\hline $14 \mathrm{~h}$ & Suco de maracujá com adoçante Diet & $50 \mathrm{~mL}$ & 9 \\
\hline $16 \mathrm{~h}$ & Gelatina Diet & $50 \mathrm{~mL}$ & 15 \\
\hline $18 \mathrm{~h}$ & Sopa líquida restrita & $50 \mathrm{~mL}$ & 25 \\
\hline $20 \mathrm{~h}$ & Suco de laranja coado e sem adoçar & $50 \mathrm{~mL}$ & 32 \\
\hline \multicolumn{4}{|c|}{ Segundo dia de dieta pós-gastroplastia ( $\left.2^{\circ} \mathrm{PO}\right)$} \\
\hline Horário & Alimento & Quantidade & Kcal \\
\hline $8 \mathrm{~h}$ & Leite desnatado sem adoçar & $50 \mathrm{~mL}$ & 18 \\
\hline $10 \mathrm{~h}$ & Gelatina Diet & $50 \mathrm{~mL}$ & 15 \\
\hline $12 \mathrm{~h}$ & Sopa liquidificada coada & $50 \mathrm{~mL}$ & 25 \\
\hline $14 \mathrm{~h}$ & Chá de ervas adoçado com 1 colher (sobremesa) de açúcar & $50 \mathrm{~mL}$ & 1 \\
\hline $16 \mathrm{~h}$ & Pudim Diet sem calda & $50 \mathrm{~mL}$ & 20 \\
\hline $18 \mathrm{~h}$ & Sopa liquidificada coada & $50 \mathrm{~mL}$ & 25 \\
\hline $20 \mathrm{~h}$ & Suco de laranja coado & $50 \mathrm{~mL}$ & 32 \\
\hline
\end{tabular}


tenha apresentado boa tolerância à alimentação no primeiro dia.

Para o cálculo de energia oferecidas diariamente no hospital foi utilizada a Tabela de Composição Química dos Alimentos do autor Guilherme Franco, 1997. Observa-se a oferta de aproximadamente $140 \mathrm{kcal} / \mathrm{dia}$ nos dois primeiros dias de alimentação. É sugerido ao paciente que leve água de coco na internação e que esta seja ingerida em pequenos goles, totalizando um volume mínimo de $500 \mathrm{~mL}$ durante o dia, o que faz com que o consumo energético mínimo do paciente seja de $240 \mathrm{kcal} / \mathrm{dia}$.

A alta hospitalar ocorre geralmente no terceiro dia pós-operatório, o qual coincide com o segundo dia de alimentação. Quando o paciente permanece internado por um período maior, segue-se o mesmo esquema do segundo dia, com modificações apenas nos ingredientes das sopas e sucos, com a finalidade de variar o sabor das preparações.

\section{No pós-operatório}

Enfatiza-se a importância do Aconselhamento Nutricional cuidadoso no pós-operatório, pois o paciente passará por inúmeras modificações de hábitos alimentares. Segundo Antonini et al. ${ }^{13}$, somente o acompanhamento nutricional adequado garante o sucesso da cirurgia, evitando complicações, como vômitos, intolerância alimentar e perda de peso insuficiente.

Após a alta hospitalar, o paciente segue um plano gradual de reintrodução de alimentos, com um consumo energético inicial de $300 \mathrm{kcal} / \mathrm{dia}$ a $350 \mathrm{kcal} / \mathrm{dia}$, atingindo aproximadamente $700 \mathrm{kcal}$ na terceira semana, conforme cálculo feito por meio da Tabela de Composição Química dos Alimentos do autor Guilherme Franco ${ }^{14}$. Durante a primeira semana o paciente recebe uma dieta líquida completa, com um volume de $50 \mathrm{~mL}$ por refeição, totalizando 8 refeições ao dia. Na segunda semana, há evolução para consistência pastosa com um volume máximo de $100 \mathrm{~mL}$ e, na terceira semana, é introduzida a alimentação de consistência normal, com volume máximo de $150 \mathrm{~mL}$ por refeição. Como citado anteriormente, estas orientações são fornecidas ao paciente durante o pré-operatório.

Durante as consultas de pós-operatório, salienta-se a necessidade de atenção constante quanto à mastigação até que o alimento se torne pastoso na boca, bem como quanto à importância de não consumir maior quantidade de alimentos do que a recomendada, ou seja, $50 \mathrm{~mL}$ de duas em duas horas de alimentos líquidos e coados durante a primeira semana, $100 \mathrm{~mL}$ de alimentos pastosos durante a segunda semana e $150 \mathrm{~mL}$ ou 3 colheres de sopa de alimentos sólidos durante a terceira semana, para evitar complicações como vômitos ou obstrução, e de fazer as refeições em ambiente calmo, tranqüilo e nunca apressadamente. Para garantia de uma boa hidratação, alerta-se sobre a vigilância quanto à ingestão de líquidos, em pequenas quantidades, nos intervalos entre as refeições e nunca durante, para evitar dilatação do estômago e interferência no processo digestivo, dando preferência à água de coco e às bebidas isotônicas, devido ao aporte de vitaminas e minerais. Além disso, recomenda-se que, quando consumidos alimentos ou bebidas muito quentes ou muito geladas, estes sejam mantidos por um tempo suficiente na boca, para que haja adequação da temperatura antes da deglutição.

Sugere-se que os pacientes tenham copos e xícaras pequenos em casa, além de utilizarem o prato de sobremesa para fazerem as suas refeições, o que possibilita uma melhor apresentação visual da refeição, quando comparada à distribuição de alimentos em pratos e copos de tamanho padrão.

No momento da evolução do esquema alimentar de uma semana para a outra, o paciente é orientado a observar se terá condições de consumir a quantidade indicada, maior que a da semana anterior. Recomenda-se um cuidado especial na primeira refeição. Caso esteja farto antes de consumir a quantidade indicada, é 
orientado a não forçar, evitando vômitos. O paciente deve estar ciente também de que pode haver variações de consumo de uma refeição para a outra e que o volume indicado é o máximo que ele pode consumir, não devendo preocupar-se caso consuma menos, esporadicamente.

Na terceira semana, com o início da alimentação de consistência normal, ressalta-se a necessidade de especial atenção quanto à introdução de carnes e vegetais crus, devido à freqüente intolerância a esses alimentos, observada na prática clínica. São evitados, no primeiro dia de dieta, carne vermelha e vegetais crus, devendo ser realizada uma evolução gradual durante a terceira semana, conforme a tolerância. Alimentos causadores de flatulência são evitados inicialmente, assim como bebidas gaseificadas, doces e gorduras. O paciente é orientado quanto à possibilidade de apresentar intolerância à carne vermelha, ao leite (principalmente integral) e, com menos freqüência, arroz ou pão.

Após 21 dias de pós-operatório, o paciente deverá consultar o serviço de nutrição, que fará nova avaliação nutricional, verificando as alterações de hábitos alimentares, intolerâncias alimentares, peso perdido, presença de vômitos e diarréia ou constipação.

Com esta avaliação deverá ser feita também uma nova proposta alimentar, de acordo com as condições clínicas e socioeconômicas do paciente, além de ser iniciada a suplementação de vitaminas e minerais, devido à baixa ingestão alimentar. Esta só não é iniciada anteriormente devido à dificuldade para ingestão de comprimidos e intolerância quando estes são ingeridos macerados ou através de compostos líquidos, devido ao sabor amargo que apresentam; porém, quando houver tolerância, deverá ser iniciada o mais breve possível.

A partir desse momento, o paciente deverá comparecer a consultas com o nutricionista, mensalmente, para dar continuidade ao tratamento. Verifica-se a necessidade de acompanhamento constante, pois durante as consultas itens essenciais para evitar sintomas indesejáveis e complicações são monitorados, verificando-se a necessidade de suplementos específicos e discutindo-se as modificações necessárias na alimentação, de acordo com a tolerância de cada um. O procedimento nas consultas deve incluir recordatório de 24 horas e a freqüência alimentar, avaliando-se os alimentos consumidos para garantia de ingestão mínima de $40 \mathrm{~g}$ a $60 \mathrm{~g}$ de proteínas por dia ${ }^{15}, 1200 \mathrm{~mL}$ de líquidos e as necessidades de vitaminas e minerais, segundo as Ingestões Dietéticas de Referência (DRI), verificado por meio de análise química da dieta com utilização de programas específicos de software para garantia do aporte necessário de micronutrientes, pela alimentação ou pela suplementação enquanto for necessário. Verifica-se, também, a mastigação, a presença de intolerâncias alimentares, mudanças no funcionamento intestinal e a freqüência de náuseas e vômitos.

\section{RESULTADOSE DISCUSSÃO}

Quanto a redução de peso no pré-operatório, 15 dias após a primeira consulta, foi constatado que $60 \%$ dos pacientes perderam $2 \mathrm{~kg}$ neste período; $30 \%$ mantiveram seu peso e 10\% ganharam $0,6 \mathrm{~kg}$.

Por meio destes resultados pode-se verificar a capacidade de adesão às orientações nutricionais estabelecidas, sendo este um fator essencial à evolução no pós-operatório. A mudança de hábitos - incluindo melhor mastigação, horários fixos para refeições, fracionamento adequado e ingestão de vegetais e frutas, assim como redução do consumo de alimentos ricos em gorduras e açúcares simples - é a principal meta a ser alcançada pelo paciente no pré-operatório. Essas medidas irão facilitar a aceitação da dieta pós-operatória, reduzindo as possibilidades de complicação, já que o paciente estará preparado para as mudanças que deverão ocorrer.

A freqüência de vômitos até o $21^{\circ}$ dia de alimentação no pós-operatório foi a seguinte: 
46,70\% dos pacientes não apresentaram vômitos; 16,66\% apresentaram 1 episódio de vômito; 13,33\% apresentaram 2 episódios; e 23,33\% apresentaram 3 ou mais episódios neste período, não havendo necessidade de hospitalização em nenhum dos casos.

Martin et al. ${ }^{11}$ analisaram as complicações pós-operatórias de 100 pacientes dos quais 8,0\% apresentaram vômitos que necessitaram de hospitalização devido à desidratação. Mottin et al. ${ }^{16}$ acompanharam 149 pacientes, dos quais 48,9\% apresentaram vômitos sobretudo no $2^{\circ}$ mês de pós-operatório, coincidindo com a introdução de alimentos de consistência normal, relatando que este foi mais prevalente com a ingestão de carne e arroz.

Considerou-se a análise da freqüência de vômitos no pós-operatório como indicativo de adesão ao protocolo proposto por serem atribuídos, com raras exceções, à ingestão de alimentos gordurosos ou doces concentrados, mastigação insuficiente, ingestão demasiada ou alimentação realizada em curto espaço de tempo $0^{3,10,16}$. Todos os pacientes que apresentaram episódios de vômito tiveram a capacidade de relatar à nutricionista o motivo da ocorrência do problema. Este fato demonstra a importância da orientação sobre fatores que causam vômitos e formas de prevenção, incluídos no protocolo do período pré-operatório.

As principais intolerâncias alimentares relatadas pelos pacientes até o $21^{\circ}$ dia de alimentação no pós-operatório foram: 53,53\% dos pacientes não apresentaram intolerâncias alimentares; 23,33\% tiveram intolerância à carne; 3,33\%, ao arroz; 3,33\%, ao pão e 13,33\%, a mais de um alimento. Neste período não ingeriram frituras, alimentos gordurosos e doces concentrados.

Nelson et al. ${ }^{3}$ descrevem a ocorrência de intolerância à carne vermelha e ao leite, levando a mal-estar e náuseas. A experiência negativa com o alimento faz com que os pacientes tenham receio de ingeri-lo novamente, retardando a sua introdução. A orientação no pré-operatório, quanto à possibilidade de ocorrência de desconforto na introdução destes alimentos e sobre a necessidade de introdução gradual, reduziu a freqüência de ocorrência do problema.

Quanto a porcentagem de peso perdido até $021^{\circ}$ dia de alimentação no pós-operatório, em relação ao peso na ocasião da cirurgia (Figura 1), verifica-se que $76,7 \%$ dos pacientes perderam mais de $10,0 \%$ de seu peso no período; $13,3 \%$ perderam entre $9,5 \%$ e $10,0 \%$ de seu peso e apenas $10,0 \%$ perderam entre $8,0 \%$ e $9,5 \%$ de seu peso. Os resultados demonstram uma redução de peso equivalente à encontrada em literatura após o primeiro mês de cirurgia, que foi de $8,6 \%$ para Arazaki et al. ${ }^{17}, 10,8 \%$ de acordo com Lima et al. ${ }^{18}$ e 9,1 \% segundo Garrido Júnior et al. ${ }^{1}$.

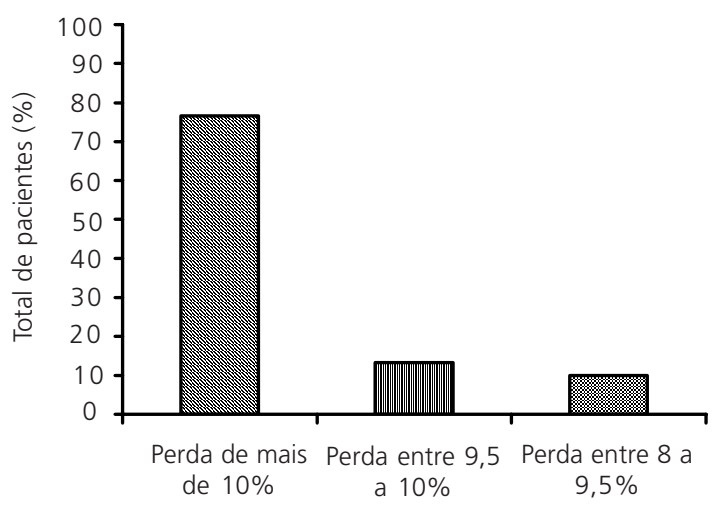

Figura 1. Perda de peso em relação ao peso apresentado no pré-operatório até $021^{\circ}$ dia de alimentação pós-operatória.

Os resultados deste estudo demonstram que o protocolo de atendimento nutricional proposto, diferenciado do recomendado em literatura quanto ao trabalho realizado durante o período pré-operatório e evolução para dieta de consistência normal em 15 dias, permitiu a adesão dos pacientes observada pela baixa incidência de complicações de vômitos e intolerâncias alimentares, e pela perda de peso equivalente à relatada em literatura. Esses resultados permitem ressaltar a importância da atuação do profissional nutricionista como educador na equipe de trabalho para atendimento ao paciente em tratamento cirúrgico de obesidade mórbida. 
Knaane \& Ortigoso ${ }^{19}$ destacam que os pacientes são diferentes uns dos outros e que o comportamento humano não é determinado por leis biológicas, mas por leis complexas relativas às ciências humanas em que o individual, o único deve ser considerado. Dentro desta premissa, a habilidade dos profissionais que lidam com o obeso mórbido está em entender os múltiplos aspectos de vida que o envolvem para poder auxiliá-lo efetivamente. A confiança mútua e o canal aberto para diálogo, além da troca de informações entre os membros da equipe multiprofissional, tiveram indiscutivelmente um papel-chave no sucesso do protocolo proposto.

\section{O N CLUSÃ O}

Em conclusão, o protocolo proposto trouxe resultados adequados no sentido de prevenir as principais complicações no pós-operatório e de produzir resultados favoráveis quanto à perda de peso, no período que compreende o preparo para a cirurgia até $o 21^{\circ}$ dia de pós-operatório.

Os resultados demonstram que a evolução de consistência da dieta a cada 7 dias nas três primeiras semanas de pós-operatório, quando precedida por um trabalho cuidadoso de orientação e conscientização do paciente, iniciado no pré-operatório, melhora a adesão à terapia nutricional no pós-operatório, devido à reintrodução precoce de alimentos sólidos, o que evita a monotonia alimentar de dietas de consistência líquida e pastosa, estimulando a ingestão de alimentos. Esta melhoria é obtida sem o aumento de incidência de complicações pósoperatórias. Além disso, possibilita ao paciente retornar às suas atividades habituais sem necessitar de uma alimentação de consistência modificada, tendo apenas a especificidade da escolha de alimentos com baixos teores de gorduras e açúcares simples e do controle de volumes ingeridos.

Enfatiza-se com este trabalho a importância da elaboração de um protocolo de atendimento nutricional que contemple e priorize aspectos de educação nutricional, preparo cuidadoso do paciente no pré-operatório e monitorização detalhada no pós-operatório. Recomenda-se que o protocolo de atendimento nutricional seja utilizado como ferramenta para a construção do trabalho do nutricionista, porém deve-se ressaltar que cada paciente deve ser considerado um ser único. Assim, no momento do aconselhamento nutricional, o tempo necessário para a compreensão do paciente sobre todas as orientações, para adaptação das metas estabelecidas ao seu ritmo de vida, e para evolução da dieta de uma consistência a outra deve ser individualizado e suficiente para proporcionar a criação de uma relação de confiança mútua entre nutricionista e paciente.

Para maximização dos resultados da cirurgia bariátrica é necessária a formação de uma equipe multidisciplinar coesa, em que todos os membros cheguem a um consenso quanto às condições psicológicas, nutricionais e de saúde do paciente, indicando ou não a cirurgia. A equipe deve estar em constante interação para troca de informações e consciente de que o trabalho isolado não surte o efeito desejado por não considerar todos os aspectos do indivíduo sendo, por isso, essenciais a avaliação e a atuação de todos os profissionais da equipe, monitorando o paciente como um todo desde o pré-operatório.

Há carência de publicações sobre o tratamento cirúrgico de obesos mórbidos relacionados diretamente à área de nutrição, motivo pelo qual se espera que outros grupos de tratamento ao obeso mórbido divulguem os resultados do trabalho desenvolvido para enriquecimento mútuo.

Objetiva-se a continuidade do presente estudo, avaliando-se o estado nutricional, as condições de alimentação do paciente a médio e longo prazo e os resultados dos exames laboratoriais para verificação de adequações necessárias no protocolo de atendimento e para estabelecimento de diretrizes de conduta nutricional eficazes no pós-operatório tardio. 


\section{REFERÊ NCIAS}

1. Garrido Jr AB, Halpern A, Rodrigues JJG. Cirurgia para obesidade grave: vivência de duas décadas. Rev Bras Nutr Clín 2000; 15(3):400-5.

2. Shikora SA. Surgical treatment for severe obesity: the state-of-the-art for the new millennium. Nutr Clin Pract 2000; 15(1):13-22.

3. Nelson JK, Gastineau CF, Moxness KE. Mayo clinic diet manual: a handbook of nutrition practices. Missouri: Mosby; 1994. p.195-205.

4. Fisher BL, Barber AE. In: Deitel M, Cowan Jr GSM. Update: surgery for the morbidly obese patient. Canada: FD-Communications; 2000. p.139-44.

5. Herrera MF, Lozano-Salazar RR, Gonzáles-Barranco J, Rull JA. In: Deitel M, Cowan Jr GSM. Update: surgery for the morbidly obese patient. Canada: FD Communications; 2000. p.55-62.

6. Csendes A, et al. Resultados preliminares de la gastroplastia horizontal con anastomosis en $\mathrm{Y}$ de Roux como cirurgia bariática en pacientes con obsidad severa y mórbida. Rev Méd Chil 1999; 127(8): 953-60.

7. Cowan GSM, Buffington CK. Significant changes in blood pressure, glucose, and lipids with gastric bypass surgery. World J Surg 1998; 22:987-92.

8. Valezi AC, Brito EM, Souza JCL, Liberatti M, Marson AC, Bocardo SD. Cirurgia de Capella - experiência de 200 casos. Bol Cirur Obes 2001; 2(4):3.

9. Halverson JD. Micronutrient deficiencies after gastric bypass for morbid obesity. Am Surg 1986; 52(11):594-8.

10. Cronin BS, Mac Donough AB. Nutrition management of morbid obesity in conjunction with surgical intervention. Top clin nutr 1987; 2(2):59-68.

11. Martin LF, et al. Preoperative Insurance Status Influences Postoperative Complication Rates for Gastric Bypass. Am J Surg 1991; 161(6):625-43.

12. Martins C, Pierosan S. Manual do Educador. Curitiba: Nutroclínica; 1997.

13. Antonini DR, Pariera CRV, Simões N, Concon Filho A. Avaliação nutricional dos pacientes submetidos à cirurgia bariátrica. Bol Cirur Obes 2001; 2(4):3.

14. Franco G. Tabela de composição química dos alimentos. São Paulo: Atheneu; 1997.

15. Grace DM. Metabolic complication following gastric restrictive procedures. In: Deitel M. Surgery for the obese pacient. Portland: Bookmens; 1989. p.339-50.

16. Mottin CC, et al. Tolerância alimentar no acompanhamento pós-operatório da cirurgia bariátrica: um estudo de 149 pacientes obesos mórbidos. Bol Cirurgia da Obes 2002; 3(3):45.

17. Arasaki $\mathrm{CH}$, et al. Cirurgia de obesidade mórbida pela técnica de Capella via aberta: resultados em 100 pacientes. Bol Cirur Obes 2001; 2(4):3.

18. Lima S, Castro B, Pacheco MG, Laurino RM, Buchmann AACM, Toschi AP. Tratamento cirúrgico da obesidade mórbida: resultados em um hospital comunitário. Bol Cirur Obes 2002; 3(3):33.

19. Knaane R, Ortigoso SAF. O profissional da saúde e o seu cliente. Rev Banas Qualidade 1999; 8(89): 74-8.

Recebido para publicação em 27 de março de 2002 e aceito em 18 de junho de 2003. 